\title{
203. APORTACIONES A LA FLORA VASCULAR DE CÓRDOBA Y ANDALUCÍA OCCIDENTAL (ESPAÑA)
}

\author{
Jesús M. MUÑOZ ÁLVAREZ ${ }^{1 *}$ y María L. DÍAZ IGLESIAS ${ }^{2}$
}

Recibido el 24 de junio de 2016, aceptado para su publicación el 6 de septiembre de 2016

Contributions to the vascular flora of Cordoba and Western Andalusia (Spain)

Palabras clave: flora vascular, nuevas citas, Andalucía, Córdoba

Key words: vascular flora, new records, Andalusia, Cordoba

Se citan por primera vez para la provincia de Córdoba cinco especies de plantas vasculares, una de ellas - Barbarea vernanovedad, además, para Andalucía Occidental. Se aporta igualmente una segunda cita de Corynephorus canescens para Córdoba. Para la identificación de los taxones se han utilizado fundamentalmente Flora Iberica (Castroviejo, 1986-2012), Flora Vascular de Andalucía Occidental (Valdés et al., 1987) y Flora Vascular de Andalucía Oriental (Blanca et al., 2009), y para las Gramíneas se ha seguido a Romero Zarco (2015). El material herborizado se encuentra depositado en el Herbario de la Facultad de Ciencias de Córdoba (COFC). Para cada especie, junto a la o las localidades concretas, se presenta un análisis corológico centrado en el territorio, así como información sobre el hábitat en el que se han recolectado.

Barbarea verna (Mill.) Asch., Fl. Brandenburg 1: 36 (1860)

Erysimum vernum Mill., Gard. Dict. ed. 8, n. 2 (1768)

Córdoba. Córdoba, Sierra Morena, río Guadiato, 260 m, [30SUH3301], 14-04-2014, J.M. Muñoz, (COFC 62407).

Hábitat: depósitos fluviales arenosos y húmedos, en las inmediaciones de cursos de agua, en zonas temporalmente inundadas.
Crece en comunidades abiertas, junto con Equisetum ramosissimum Desf., Ranunculus muricatus L., Geranium purpureum Vill., Rumex conglomeratus Murray, etc.

Especie poco frecuente en Andalucía. Para Andalucía Oriental fue citada -sub Barbarea praecox- por Willkomm \& Lange (1880) y Boissier (1839) de Sierra Nevada, donde fue indicada nuevamente por Molero Mesa \& Pérez Raya (1987). Más recientemente, Fernandes (1993) la indica solo de Granada y Almería, y Morales Torres (2009) para el complejo Nevada-Filabres y las Alpujarras. Clemente Muñoz \& Hernández Bermejo (1987) no recogen la especie para la flora de Andalucía Occidental, por lo que su hallazgo en Córdoba constituye probablemente la primera cita para este territorio.

Beta macrocarpa Guss., Fl. Sic. Prod. 1: 302 (1827)

Córdoba. Baena, Albendín, laguna de Consuegra, 368 m, [30SUG9272], 16-07-2014, M.L. Díaz \& J.M. Muñoz, (COFC 62409). Lucena, laguna Albina, 409 m, [30SUG5729], 23-07-2014, M.L. Díaz \& J.M. Muñoz, (COFC 62410).

Hábitat: se la ha encontrado en terrenos removidos por el arado, en las zonas de transición entre los suelos inundados estacionalmente y los herbazales densos que 
suelen dar ya paso a las tierras cultivadas (olivar fundamentalmente, girasol, etc.). Forma parte de comunidades terofíticas halonitrófilas, de pequeña talla y con una cobertura del $70-75 \%$, que forman una cubierta vegetal discontinua, en ocasiones asociada a los valles de los surcos hechos por el arado. Se trata de comunidades de Frankenietalia pulverulentae Rivas-Martínez ex Castroviejo \& Porta, en las que aparece junto a Frankenia pulverulenta L., Polypogon maritimus Willd., Spergularia nicaeensis Sarato ex Burnat, Spergularia heldreichii Foucaud, Suaeda splendens (Pourr.) Gren. \& Godr., etc. No es infrecuente su presencia también en los herbazales densos (y altos, de más de $1 \mathrm{~m}$ ) antes mencionados, dominados por especies de Artemisietea vulgaris Lohmeyer, Preising \& Tüxen ex von Rochow (como Helminthoteca echioides (L.) Holub o Carduus bourgeanus Boiss. \& Reut.) y Stellarietea mediae Tüxen, Lohmeyer \& Preising \& ex von Rochow (como Conyza canadensis (L.) Cronq., Rapistrum rugosum (L.) All, etc.).

Taxón raro en Andalucía, donde lo menciona Gutiérrez Bustillo (1990) de Almería, Cádiz y Sevilla. Cabello (2009) indica su presencia en Andalucía Oriental para las áreas naturales de Trevenque-Almijara, Alpujarras, Almería y Axarquía; Cobo Muro (2001) lo cita también del área Guadaquivir, en Jaén, y Pastor (1987) solo lo da de las comarcas Litoral y Marisma en Andalucía Occidental. A las citas antiguas de Willkomm \& Lange (1870), Pérez Lara (1887) y Colmeiro (1888), sub B. bourgaei, de la comarca Litoral de Cádiz, cabe añadir -para esta comarca- la de Galiano \& Silvestre (1975). De la Marisma o Litoral de Huelva es citada por Rivas-Martínez et al. (1980) y de la Marisma de Sevilla por Martínez Parras \& Peinado Lorca (1993). Las localidades del sur de Córdoba, son las primeras de las que tenemos constancia para esta provincia y para las comarcas Campiña Baja y Campiña Alta en Andalucía occidental.

Corynephorus canescens (L.) P. Beauv.,
Agrost. 90: 159 (1812)

Aira canescens L., Sp. Pl. 65 (1753)

Córdoba. Córdoba, Sierra Morena, inmediaciones de El Lagar de la Cruz, 548 m, escorial de mina, [30SUH3901], 30-09-2015, J.M. Muñoz, (COFC 62405).

Hábitat: observada en escorias procedentes de la fundición de calcopirita de una mina abandonada, de época romana. Aparece formando una cubierta vegetal heterogénea, con predominio en unas partes de Corynephorus canescens y, en otras, de Rumex bucephalophorus subsp. gallicus (Steinh.) Rech., lo que repercute en la fisionomía de la comunidad (muestra el tono rojizo característico por el predominio de la segunda especie); hay también variabilidad en el grado de cobertura de unos puntos a otros (desde 30-35\% a $65 \%$ ). No obstante dicha heterogeneidad, el cortejo florístico es bastante homogéneo, así como pauciespecífico (y coincidente en buena medida con el dado por Melendo, 1998, para la localidad de Pedroches). Con excepción de $C$. canescens y de Corrigiola telephiifolia Pourr., ambas hemicriptófitos, el resto de especies son terófitos, predominantemente de Tuberarietalia guttatae Br.-Bl. in Br.-Bl., Molinier \& WagnerLinaria spartea (L.) Chaz., R. bucepahlophorus subsp. gallicus, Micropyrum tenellum (L.) Link, Jasione montana L.- - y, en menor medida, de Polygono-Poetalia annuae Tüxen in Géhu, Richard \& Tüxen-Spergularia purpurea (Pers.) D. Don y Plantago coronopus L.- Son comunidades encuadrables en Corynephorion canescentis Klika.

La especie se conocía en Andalucía Occidental solo de las comarcas Litoral y Algeciras (Huelva y Cádiz) (Romero Zarco, 1987), hasta que en 1998 Melendo la cita por primera vez para la provincia de Córdoba, de la comarca Pedroches. Su distribución se debe ampliar también a la Campiña Baja en Cádiz (Paunero, 1956), Marisma y Litoral en Sevilla (Paunero, 1956; Garrido et al., 2002), y Andévalo y Aracena en Huelva 
(Santa-Bárbara et al., 2003; Valdés et al., 2008). La población encontrada supone la segunda cita que conocemos para Córdoba y la primera para la comarca Sierra Norte en Andalucía.

Damasonium polyspermum Coss., Notes $\mathrm{Pl}$. Crit. 47 (1849)

Córdoba. Baena, laguna de Casasola, $285 \mathrm{~m}$, [30SUG8780], 10-07-2014, J.M. Muñoz, (COFC 62408).

Hábitat: ha sido recolectada en terrenos no arados de la laguna desecada, formando parte de una comunidad abierta de terófitos efímeros (cobertura del 70\%), dominada por Heliotropium supinum L., y junto a especies como Damasonium bourgaei Coss., Verbena supina L., Lythrum tribracteatum Spreng., Lythrum acutangulum Lag. y Chrozophora tinctoria (L.) Raf.; comunidad encuadrable en la alianza Verbenion supinae Slavnic de IsoetoNanojuncetea Br.-B1. \& Tüxen ex Westhoff, Dijk \& Passchier.

Taxon poco frecuente en Andalucía Occidental, donde Talavera (1987) lo señala del Litoral de Huelva y Cádiz, de la Marisma, de la Campiña Baja gaditana y del extremo más occidental de la Vega y del Condado-Aljarafe en Sevilla. No se conoce su presencia en Córdoba (Talavera \& Talavera, 2010), por lo que se trata de la primera cita para esta provincia (Campiña Baja).

Melilotus siculus (Turra) B. D. Jacks., Index Kew. 2: 199 (1894)

Trifolium siculum Turra, Farsetia: 12 n. ${ }^{\circ}$ 5 (1765)

Córdoba. Lucena, laguna de Vadohondo, 376 m, [30SUG6032], 23-04-2015, J.M. Muñoz, (COFC 62406).

Hábitat: se ha localizado en una de las dos parcelas dedicadas al cultivo de la laguna de Vadohondo. Forma, a partes iguales con Melilotus segetalis (Brot.) Ser. in DC., poblaciones continuas y densas, entre las que crecen especies como Lolium rigidum Gaudin, Phalaris brachystachys Link, Polypogon maritimus, Juncus bufonius L., Melilotus indicus (L.) All., etc.

Especie poco frecuente en Andalucía Occidental, que Domínguez (1987, sub Melilotus messanensis), indica solo para las comarcas Marisma, Litoral gaditano y Campiña Baja de Cádiz y Sevilla. Para dicho territorio se conoce también de Huelva y del Litoral (Rivas-Martínez et al., 1980; Sánchez Gullón $\&$ Weickert, 2002). No había sido indicado para Córdoba por Sales \& Hedge (2000), por lo que se trata de la primera cita para esta provincia (Campiña Alta).

Spergularia heldreichii Foucaud, Note Spergularia 5 (1903)

Córdoba. Puente Genil, laguna de Tíscar, 167 m, [30SUG3844], 23-05-2014, J.M. Muñoz, (COFC 62412). Lucena, laguna Albina, $409 \mathrm{~m}$, [30SUG5729], 23-07-2014, M.L. Díaz \& J.M. Muñoz, (COFC 62411).

Hábitat: en la laguna Albina comparte el mismo hábitat que Beta macrocarpa, esto es comunidades terofíticas halonitrófilas de Frankenietalia pulverulentae, dominadas por Spergularia tangerina P. Monnier, junto a Frankenia pulverulenta, Polypogon maritimus y Juncus bufonius, y en herbazales de transición a la zona cultivada. En la laguna de Tíscar se ha observado en suelos arcillosos ligeramente agrietados, en comunidades abiertas dominadas por Frankenia laevis L., junto a Cressa cretica L., Polypogon maritimus y Pulicaria paludosa Link.

Especie poco común en Andalucía. Díaz de la Guardia (2009) la cataloga de rara en Andalucía oriental y menciona su presencia solo en las áreas naturales Guadalquivir y Ronda. En Andalucía Occidental, Devesa (1987) la da únicamente de la comarca Litoral. Tampoco se aportan localidades para Sevilla y Córdoba en Anthos (2016), aunque Ratter (1990) sí incluye Sevilla en su área de distribución, presencia atestiguada también por Lendínez et al. (2011). Candau \& Devesa (1983) y Devesa (1983) la dan por primera vez 
para la comarca de Grazalema. La cita aquí indicada constituye novedad para la provincia de Córdoba (Campiña Alta).

\section{BIBLIOGRAFÍA}

ANTHOS -2016- Sistema de información sobre las plantas de España. www.anthos.es (Fecha de acceso: 2016-04-15).

BLANCA, G., B. CABEZUDO, M. CUETO, C. FERNÁNDEZ LÓPEZ \& C. MORALES TORRES (eds.). -2009- Flora Vascular de Andalucía Oriental, 4 vols. Consejería de Medio Ambiente, Junta de Andalucía, Sevilla.

BOISSIER, E. -1839-Voyage botanique dans le midi de L'Espagne pendant l'anne 1837. Vol. II, Paris, Gide et Cie., Libraires-editeurs.

CABELlO, J. -2009- Beta L. En G. Blanca, B. Cabezudo, M. Cueto, C. Fernández Lopez \& C. Morales Torres (eds.). Flora Vascular de Andalucía Oriental 2: 157. Consejería de Medio Ambiente, Junta de Andalucía, Sevilla.

CANDAU, P. \& J.A. DEVESA -1983- Contribución al conocimiento del polen y de las semillas del género Spergularia en Andalucía occidental. Lazaroa 5: 187-200.

CASTROVIEJO, S. (coord. gen.). -1986-2012- Flora Ibérica 1-8, 10-15, 17-18, 21. Real Jardín Botánico, CSIC, Madrid.

CLEMENTE MUÑOZ, M. \& J.E. HERNÁNDEZ BERMEJO -1987- Barbarea R. Br. in W. T. Aiton. En B. Valdés, S. Talavera \& E. FernándezGaliano (eds.). Flora Vascular de Andalucía Occidental 1: 390-391. Editorial Ketres, Barcelona.

COBO MURO, M.C. -2001- Ecología y distribución de taxa y sintaxa vegetales en los ambientes halofíticos del Alto valle del Guadalquivir. Bol. Inst. Est. Giennenses 179: 219-265.

COLMEIRO, M. -1888- Enumeración y revisión de las plantas de la Península hispano-lusitana e Islas Baleares. 4. Madrid.

DEVESA, J.A. -1983- 70. Spergularia heldreichii. Lagascalia 11: 107.

DEVESA, J.A. -1987- Spergularia (Pers.) J. \& C. Presl. En B. Valdés, S. Talavera \& E. FernándezGaliano (eds.). Flora Vascular de Andalucía Occidental 1: 218-223. Editorial Ketres,
Barcelona.

DÍAZ DE LA GUARDIA, C. -2009- Spergularia (Pers.) J. Presl \& C. Presl. En G. Blanca, B. Cabezudo, M. Cueto, C. Fernández Lopez \& C. Morales Torres (eds.). Flora Vascular de Andalucía Oriental 2: 88-91. Consejería de Medio Ambiente, Junta de Andalucía, Sevilla.

DOMÍNGUEZ, E. -1987- Melilotus Miller. En B. Valdés, S. Talavera \& E. Fernández-Galiano (eds.). Flora Vascular de Andalucía Occidental 2: 129-132. Editorial Ketres, Barcelona.

FERNANDES, R.B. -1993- Barbarea R. Br. En S. Castroviejo, C. Aedo, C. Gómez Campo, M. Laínz, P. Monserrat, R. Morales, F. Muñoz Garmendia, G. Nieto Feliner, E. Rico, S. Talavera \& L. Villar (eds.). Flora Ibérica 4: 98-101. Real Jardín Botánico, C.S.I.C., Madrid.

GALIANO, E.F. \& S. SILVESTRE -1975- Catálogo de las plantas vasculares de la provincia de Cádiz II. Polygonaceae-Amaranthaceae. Lagascalia 5: 85-112.

GARRIDO, B., A. APARICIO, C. PÉREZ PORRAS, J. APARICIO, F. GARCIA MARTÍN, L. FERNÁNDEZ CARRILLO \& M.A. CARRASCO -2002- Flora de interés en Bosques-Isla de Andalucía Occidental. Acta Bot. Malacitana 27: 295-332.

GUTIÉRREZ BUSTILLO, A.M. -1990- Beta L. En S. Castroviejo, M. Laínz, G. López González, P. Monserrat, F. Muñoz Garmendia, J. Paiva, J. \& L. Villar (eds.). Flora Ibérica 2: 479-482. Real Jardín Botánico, C.S.I.C., Madrid.

LENDÍNEZ, M.L., F.M. MARCHAL \& C. SALAZAR -2011- Estudio florístico de los medios húmedos salinos de Andalucía (S. España). Catálogo y análisis de la flora vascular halófila. Lagascalia 31: 77-130.

MARTÍNEZ PARRAS, J.M. \& M. PEINADO LORCA -1993- Vegetación de los arrozales de las Marismas del Guadalquivir. Lagascalia 17: 21-35.

MELENDO LUQUE, M. -1998- De Mariani montis plantis notulae, II. Lazaroa 19: 173-178.

MOLERO MESA, J. \& F. PÉREZ RAYA -1987- $L a$ flora de Sierra Nevada. Universidad de Granada. Excma. Dip. Prov. Granada.

MORALES TORRES, C. -2009- Barbarea R. Br. En G. Blanca, B. Cabezudo, M. Cueto, C. Fernández Lopez \& C. Morales Torres (eds.). Flora Vascular de Andalucia Oriental 3: 91. Consejería 
de Medio Ambiente, Junta de Andalucía, Sevilla. PASTOR, J. -1987- Beta L. En B. Valdés, S. Talavera \& E. Fernández-Galiano (eds.). Flora Vascular de Andalucía Occidental 1: 182-183. Editorial Ketres, Barcelona.

PAUNERO, E. -1956- Las Aveneas españolas. I. Anal. Inst. Bot. Cavanilles 13: 149-229.

PÉREZ LARA, J.M. -1887- Florula gaditana. Pars secunda. Anal. Soc. Esp. Hist. Nat. 16: 273-372.

RATTER, J.A. -1990- Spergularia (Pers.) J. Presl \& C. Presl. En S. Castroviejo, M. Laínz, G. López González, P. Monserrat, F. Muñoz Garmendia, J. Paiva \& L. Villar (eds.). Flora Ibérica 2: 149-161. Real Jardín Botánico, C.S.I.C., Madrid.

RIVAS-MARTINEZ，S., M. COSTA, S. CASTROVIEJO \& E. VALDÉS -1980Vegetación de Doñana (Huelva, España). Lazaroa 2: 5-189.

ROMERO ZARCO, C. -1987- Corynephorus Beauv. En B. Valdés, S. Talavera \& E. FernándezGaliano (eds.). Flora Vascular de Andalucía Occidental 3: 333-335. Editorial Ketres, Barcelona.

ROMERO ZARCO, C. -2015- Las Gramineas de la península Ibérica e Islas Baleares. Claves ilustradas para la determinación de los géneros $y$ catálogo preliminar de las especies. Jolube Consultor Botánico y Editor, Huesca.

SALES, F. \& I.C. HEDGE -2000 - Melilotus Mill. En S. Talavera, C. Aedo, S. Castroviejo, A. Herrero, C. Romero Zarco, F.J. Salgueiro \& M. Velayos (eds.). Flora Ibérica 7(2): 720-731. Real Jardín Botánico, C.S.I.C., Madrid.

SÁNCHEZ GULLÓN, E. \& P. WEICKERT -2002Plantas del herbario de D. Bruno Weickert herborizadas en la provincia de Huelva entre los años 1950/1974. Lagascalia 22: 172-179.

SANTA-BÁRBARA, C., B. VALDÉS \& F.J. PINA -2003- 90. Novedades corológicas para la comarca del Andévalo (provincia de Huelva, SO de España). IV. Acta Bot. Malacitana 28: 237-246.

TALAVERA, S. -1987- Damasonium Mill. En B. Valdés, S. Talavera \& E. Fernández-Galiano (eds.). Flora Vascular de Andalucía Occidental 3: 183-184. Editorial Ketres, Barcelona.

TALAVERA, S. \& M.M. TALAVERA -2010Damasonium Mill. En S. Talavera, M.J. Gallego, C. Romero Zarco \& A. Herrero (eds.). Flora Ibérica 17: 18-22. Real Jardín Botánico, C.S.I.C.,
Madrid.

VALDÉS, B., S. TALAVERA \& E. FERNÁNDEZGALIANO (eds.). -1987- Flora Vascular de Andalucía Occidental, 3 vols. Editorial Ketres, Barcelona.

VALDÉS, B., C. SANTA-BÁRBARA, C. VICENT \& A. MUÑOZ -2008- Catálogo florístico del Andévalo y Sierra de Huelva (Plantas vasculares). Lagascalia 28: 117-409.

WILLKOMM, M. \& J. LANGE -1870- Prodromus florae Hispanicae, Vol. I. Stutgarttiae.

WILLKOMM, M. \& J. LANGE -1880- Prodromus florae Hispanicae, Vol. III. Stutgarttiae.

Dirección de los autores. ${ }^{1}$ Departamento de Botánica, Ecología y Fisiología Vegetal. Edificio José Celestino Mutis, Campus de Rabanales, Universidad de Córdoba, 14071 (Córdoba). ${ }^{2} \mathrm{C} /$ Donantes de Sangre n ${ }^{\circ} 32,14012$ (Córdoba). *Autor para correspondencia: bv1mualj@uco.es 